\title{
EFFECTS OF ELECTRIC FIELD AND CHARGE DISTRIBUTION ON NANOELECTRONIC PROCESSES INVOLVING CONDUCTING POLYMERS
}

\author{
Marta M. D. Ramos*, Helena M. G. Correia \\ Departamento de Física, Universidade do Minho, Campus de Gualtar, \\ 4710-057 Braga, Portugal
}

\begin{abstract}
The injection of charge carriers in conducting polymer layers gives rise to local electric fields which should have serious implications on the charge transport through the polymer layer. The charge distribution and the related electric field inside the ensemble of polymer molecules, with different molecular arrangements at nanoscale, determine whether or not intra-molecular charge transport takes place and the preferential direction for charge hopping between neighbouring molecules. Consequently, these factors play a significant role in the competition between current flow, charge trapping and recombination in polymer-based electronic devices. By suitable Monte Carlo calculations, we simulated the continuous injection of electrons and holes into polymer layers of polydiacetylene with different microstructures and followed their transport through those polymer networks. Results of these simulations provided a detailed picture of charge and electric field distribution in the polymer layer and allowed us to assess the consequences for current transport and recombination efficiency as well as the distribution of recombination events within the polymer film. In the steady state we found an accumulation of electrons and holes near the collecting electrodes giving rise to an internal electric field which is greater than the external applied field close to the electrodes and lower than that one in the central region of the polymer layer. We also found that a strong variation of electric field inside the polymer layer leads to an increase of recombination events in regions inside the polymer layer where the values of the internal electric field are lower.
\end{abstract}

\footnotetext{
* Corresponding Author: Tel:+351 253 604330; Fax:+351 253678 981; E-mail: marta@ fisica.uminho.pt
} 
Keywords: Modelling, charge distribution, internal electric field distribution, current and recombination efficiency, conducting polymer.

\section{Introduction}

Conducting polymers have received considerable attention because of their interesting electronic properties, which make them suitable to be used in electronic and optoelectronic devices [1-4] as electronically active layers with thicknesses of approximately one hundred nanometres. It has been recognized that charge transport through the polymer layer is of great importance for the operation and efficiency of these devices.

The charge transport in conducting polymers depends both on charge motion along individual molecules and on charge hopping between neighbouring molecules. Additionally, the arrangement of molecules within the polymer layer depends on the fabrication technique used. As a result, understanding and controlling the relation between charge transport and molecular arrangements (morphologies) that are obtained is crucial to modify and improve device efficiency. Therefore, the distinction between different molecular arrangements becomes most important in determining the charge distribution within the device and for the subsequent electric field distribution within the polymer layer, which affects the percolation in charge transport.

There have been experimental investigations into the internal electric fields of organic lightemitting diodes (OLED's), either using electro-absorption (EA) spectroscopy [5] or by inserting a third electrode into the bulk [6], but they can only provide average internal electric fields for a typical thickness of a polymer-based device layer. For this reason, one-dimensional device models, based on conventional band transport description, were used to calculate electric field distributions in bi-layer and multilayer OLED's [7,8]. Although the agreement 
between the simulated average electric field values obtained by these models and those obtained experimentally is good, the above models might not be adequate to obtain insights into charge and electric field distribution in polymer devices because they cannot handle properly details of the morphology of molecules within the organic layer.

Instead, we must adopt a mesoscopic approach by defining a three-dimensional arrangement of polymer molecules and rules for bipolar charge injection, transport, trapping and

recombination within the polymer layer [9]. The present work examines the charge accumulation and electric field distribution at the steady state throughout a single polymer layer comprising polydiacetylene (PDA) molecules with a Gaussian distribution of lengths. This work assesses the consequences of different molecular orientations relative to the electrode surfaces for the competition between current flow and recombination within the polymer layer.

\section{Model and simulation details}

Since the mesoscopic model of bipolar charge injection, transport and recombination in thin layers of conducting polymers used in this work has been already presented elsewhere [9], we note here only the main steps in the model. It begins with the building of PDA chain network realisations, with a density of $0.15 \mathrm{~g} / \mathrm{cm}^{3}$, between two planar electrodes separated by a distance of $100 \mathrm{~nm}$, and continues with the injection of one electron and one hole at each computer iteration (which corresponds to the time in which one inter-chain jump takes place) through PDA chains in contact with the appropriated electrodes chosen randomly. Each injected charge is then assumed to move instantaneously (i.e. before the next iteration) to the chain end which is favoured by the component of the local electric field (which is the sum of the external applied field, the field of the other charges within the polymer network and the field due to electrode polarization) along that chain if its strength is greater than $1.6 \times 10^{8} \mathrm{~V} / \mathrm{m}$ 
for holes and $2.35 \times 10^{8} \mathrm{~V} / \mathrm{m}$ for electrons, otherwise it moves towards the centre of the chain, as suggest by our previous molecular scale calculations [10].

The inter-molecular charge transport is modelled by the hopping among electronic states of neighbouring molecules. The energetic barrier depends on the difference between electron affinity (for hole transport) and ionization potential (for electron transport) of PDA molecules involved and on the potential difference caused by the local electric field. Such hopping processes are described using the generalised Monte Carlo method given in references [9] and other references therein. The data for ionization potential and electron affinities were taken from reference [7] and a dielectric constant of 2.3 used in this work was taken from reference [11].

The trapping of charge on a certain polymer chain can occur when the total electric field on the charge is zero or when the hopping rate is lower than $10^{-5}$. Finally, there is intra-molecular recombination when two charge carriers of opposite sign meet on the same PDA chain. Here we did not consider additional inter-molecular recombination.

In the calculations reported here the PDA networks were composed of straight chains, considered as rigid rods, with a Gaussian distribution of lengths and a mean length of 5 monomer units. A minimum inter-chain distance of $0.5 \mathrm{~nm}$ was used to prevent non-physical overlap of neighbour PDA chains, which are assumed to take the form $\mathrm{C}_{4 \mathrm{n}+2} \mathrm{H}_{2 \mathrm{n}+4}$. Besides, three different orientations of the molecular rods relative to the electrodes were considered: normal to electrode surfaces, parallel to electrode surfaces and randomly oriented.

\section{Results and discussion}

The bipolar charge distribution in the steady state is illustrated in figure 1 for polymer layers with the molecular rods parallel, perpendicular and randomly oriented relative to the electrode surfaces. As may be expected in absence of defective chains and impurity trapping, only a few 
percent (typically less than 5\%) of the injected charge is accumulated within the polymer layer and most electrons and holes accumulate in the region close to the collecting electrodes.

The charge accumulation is more pronounced for molecular arrangements with molecular rods perpendicular to electrode surfaces. Only for this molecular arrangement the electron accumulation dominates hole accumulation. For the other two molecular arrangements considered here, the total concentration of electrons and holes inside the polymer layer is similar but their spatial distribution along the model axis perpendicular to the electrode surfaces depends on the molecular arrangement.

The changes in the strength of the external applied electric field have little effect on the total charge accumulation. However, the distribution of the accumulated charge (electrons and holes) inside the polymer layer is sensitive to changes in the applied electric field and changes in the molecular arrangement. As the applied electric field increases, there is an increase of accumulated electrons and holes close to the collecting electrode and a decrease close to the injecting electrode for polymer chain arrangement parallel to the electrode surface. The behaviour predicted for the molecular alignment perpendicular to the electrode surface is quite different. The change in accumulated charge of both signs near the electrodes caused by a decrease in the applied electric field is negligible and the region over which electrons and holes extend into the bulk shrinks towards polymer/electrode interfaces. For the case of random oriented molecular arrangement, a change in the applied electric field mainly affects the distribution of electrons within the polymer layer but no general trend is predicted.

The electric field distribution within the polymer layer is shown in figure 2 for an external applied electric field ranging from $1 \times 10^{8} \mathrm{~V} / \mathrm{m}$ to $3 \times 10^{8} \mathrm{~V} / \mathrm{m}$. The space charge effects within the polymer layer with different molecular arrangements are quite visible here. Despite the amount of the accumulated charge within the polymer layer being very low regardless of the molecular arrangement, the space charge effect caused by electrons and holes is very pronounced contrary to the expectations. As it is easily seen in figure 2, there is a pronounced 
increase of the internal electric field at polymer-electrode interfaces which reaches values greater than the external applied electric field, whereas the electric field within the polymer layer far from the electrodes is lower than the applied field and does not change significantly through that region.

When the molecular arrangement of the polymer layer is changed, the internal electric field profile at a given applied electric field changes through the entire layer whilst the qualitative behaviour described above remains largely unchanged. For a molecular arrangement perpendicular to the electrode surface, the electric field close to the electrode where the electrons are injected is lower than the field close to the opposite electrode due to the different amount of electrons and holes accumulated close to both electrodes. The asymmetric distribution of charge accumulation of both signs is thus responsible for the asymmetric electric field profile predicted for this molecular arrangement. In polymer layers with randomly oriented molecular rods, we found also an asymmetric electric field profile mostly dictated by different distribution of electrons and holes through the polymer layer. This is in spite of the fact that their concentration is similar. The presence of symmetric distributions of electrons and holes through polymer layers with a molecular arrangement parallel to the electrode surfaces gives rise to an electric field far from the electrodes roughly homogenous. The electric field plateau is particularly pronounced at low applied electric field.

Because of strong electric field close to the electrodes, current flow perpendicular to the electrodes is enhanced in those regions. As a result most of the injected charges recombine far from the polymer/electrode interfaces. This can be seen in figure 3, where the number of recombination events as a function of position along the axis oriented normal to the electrode surfaces is shown. In this figure one may also see that recombination of electrons and holes takes place in a relatively wide region of the polymer layer which corresponds to the region where the internal electric field is lower. As the electric field decreases, the random walk of charges within the polymer layer increases. In these conditions, the probability of 
recombination increases. Therefore, recombination intensity anti-correlates with the internal electric field. This explanation holds for all molecular arrangements.

For each molecular arrangement, we found that the changes in the applied electric field made a quantitative difference to the distribution of the internal electric field within the polymer layer and consequently to the recombination intensity distribution, but qualitatively these distributions exhibit similarly shaped curves. Our results also show that when the external applied electric field increases, the recombination efficiency decreases. Since charge trapping is not significantly affected by the applied electric field, the current efficiency shows an opposite behaviour.

\section{Conclusions}

Our mesoscopic modelling has proven to be a successful method for obtaining insights into charge, electric field and recombination distribution within a polymer thin film with a specific molecular arrangement. Furthermore, when coupled to experimental data this model can aid to understanding of the observed device characteristics.

This work also shows that molecular arrangement affects mainly the distribution of accumulated charge (electrons and holes) within the polymer layer, and hence the electric field distribution through the layer, which has implications in terms of current transport and recombination efficiency, as well the recombination distribution inside the device. We found that in polymer layers with absence of defective chains or impurity trapping, the transport of injected charge towards the bulk layer is favoured by the presence of high electric field close to the electrodes and recombination events are confined to the region where the electric field is much lower.

Further studies are needed to understand the electrical properties of conducting conjugated polymer with complex nanostructures, in which ordered crystalline domains are embedded in an amorphous matrix. 


\section{Acknowledgement}

This work is part of the research project POCTI/CTM/41574/2001, approved by the Portuguese Foundation for Science and Technology (FCT) and POCTI and supported by the European Community Fund FEDER. One of us (H.M.G.C.) is also indebted to FCT for financial support under $\mathrm{PhD}$ grant $\mathrm{n}^{\circ}$. SFRH/BD/11231/2002.

\section{References}

[1] N. Sunel, A.G. Sedef, M. Parlak, and L. Toppare, Materials Chemistry and Physics 91 (2005) 227.

[2] J.H. Burroughes, D.D.C. Bradley, A.R. Brown, R.N. Marks, K. Mackay, R.H. Friend, P.L. Burns, and A.B. Holmes, Nature 347 (1990) 539.

[3] G. Yu, J. Gao, J.C. Hummelen, F. Wudl, and A.J. Heeger, Science 270 (1995) 1789.

[4] F. Garnier, R. Hajlaoui, A. Yassar, and P. Srivastava, Science 265 (1994) 1684.

[5] F. Rohlfing, T. Yamada, and T. Tsutsui, Journal of Applied Physics 86 (1999) 4978.

[6] M. Hiramoto, K. Koyama, K. Nakayama, and M. Yokoyama, Applied Physics Letters 76 (2000) 1336.

[7] S.J. Martin, G.L.B. Verschoor, M.A. Webster, and A.B. Walker, Organic Electronics 3 (2002) 129.

[8] E. Tutis, D. Berner, and L. Zuppiroli, Journal of Applied Physics 93 (2003) 4594.

[9] A.M. Stoneham, M.M.D. Ramos, A.M. Almeida, H.M.G. Correia, R.M. Ribeiro, H. Ness, and A.J. Fisher, Journal of Physics-Condensed Matter 14 (2002) 9877.

[10] M.M.D. Ramos, H.M.G. Correia, R.M. Ribeiro, and A.M. Stoneham, Synthetic Metals 147 (2004) 281.

[11] T. Hasegawa, T. Okamoto, M. Haraguchi, M. Fukui, T. Koda, and K. Takeda, Japanese Journal of Applied Physics 37 (1998) 5793. 


\section{List of figure captions}

Figure 1 - Electron and hole distribution inside the PDA network in the steady state for an external applied electric field of $3 \times 10^{8} \mathrm{~V} / \mathrm{m}$ and the following molecular orientations relative to the electrode surfaces: (a) parallel, (b) perpendicular and (c) random orientation.

Figure 2 - Internal electric field distribution inside the PDA network in the steady state calculated for the following molecular orientations relative to the electrode surfaces: (a) parallel, (b) perpendicular and (c) random orientation. Calculations are shows for an external applied electric field of $1 \times 10^{8} \mathrm{~V} / \mathrm{m}$ (squares), $2 \times 10^{8} \mathrm{~V} / \mathrm{m}$ (circles) and $3 \times 10^{8} \mathrm{~V} / \mathrm{m}$ (triangles).

Figure 3 - The calculated recombination intensity profile inside the PDA network for the following molecular orientations relative to the electrode surfaces: (a) parallel, (b) perpendicular and (c) random orientation. These results were obtained for an external applied electric field of $1 \times 10^{8} \mathrm{~V} / \mathrm{m}$ (squares), $2 \times 10^{8} \mathrm{~V} / \mathrm{m}$ (circles) and $3 \times 10^{8} \mathrm{~V} / \mathrm{m}$ (triangles). 
Figure 1
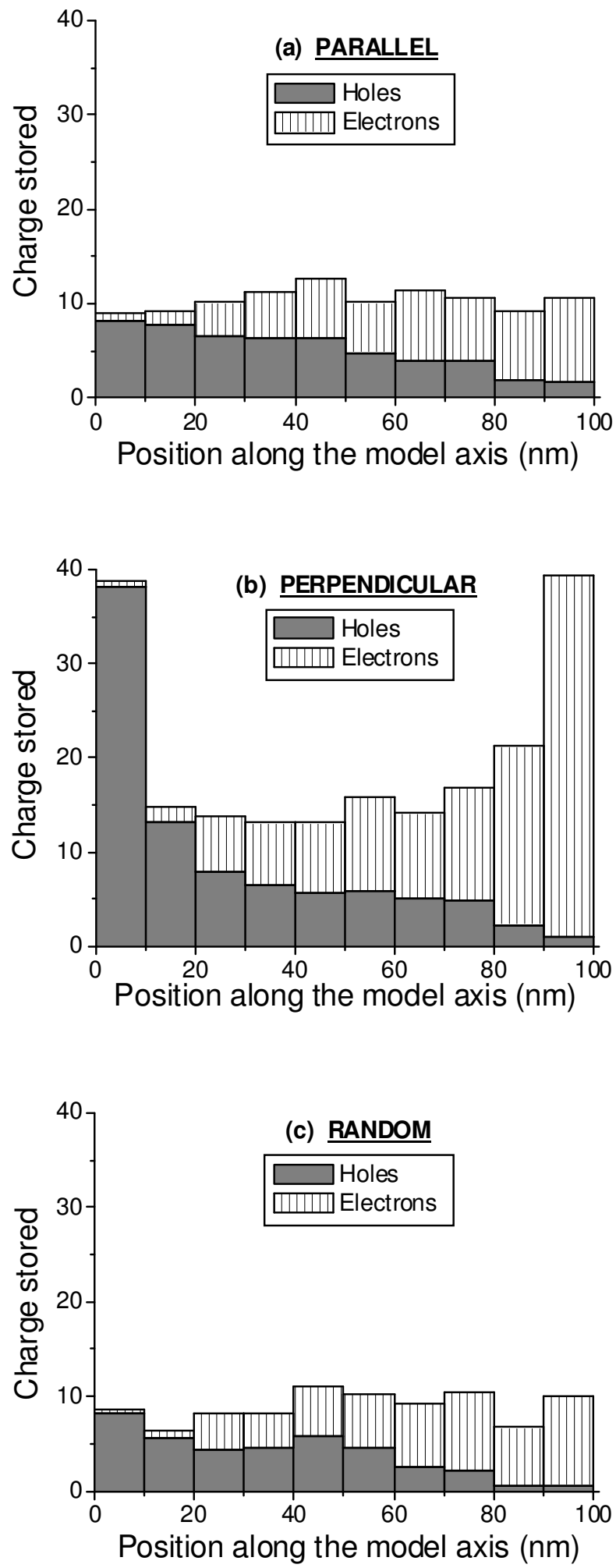
Figure 2
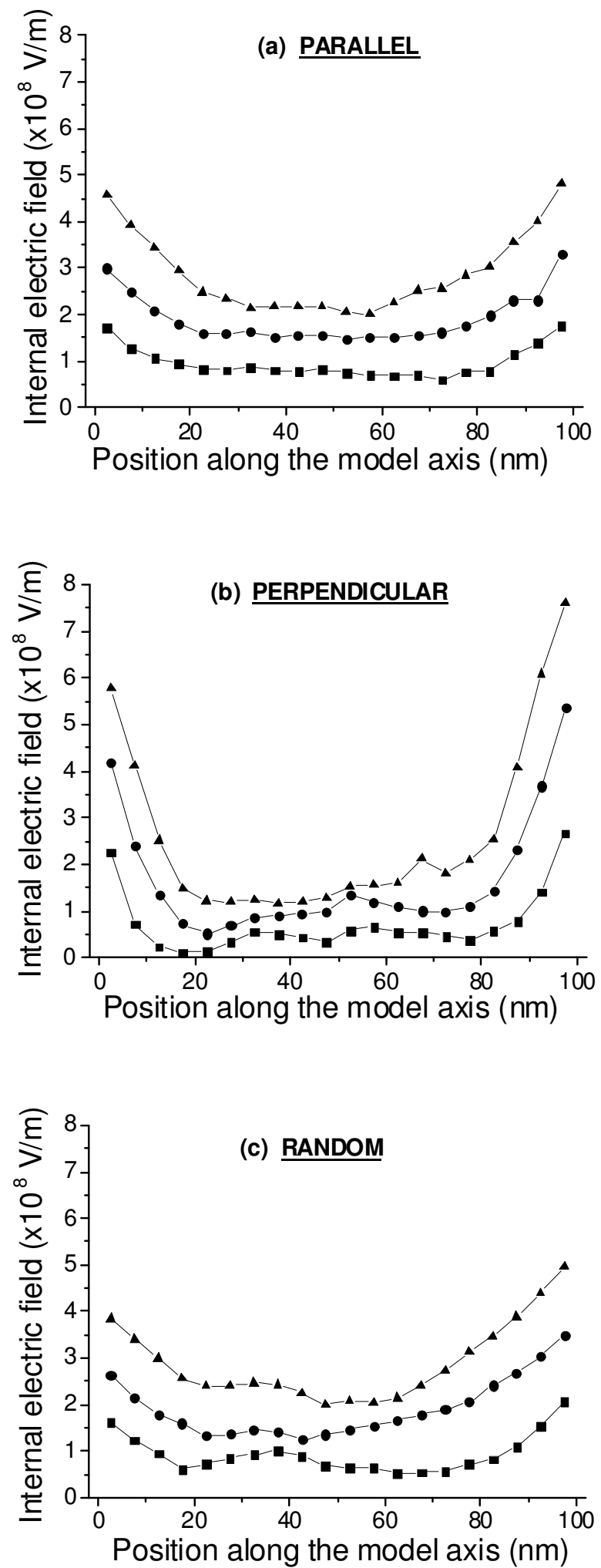
Figure 3
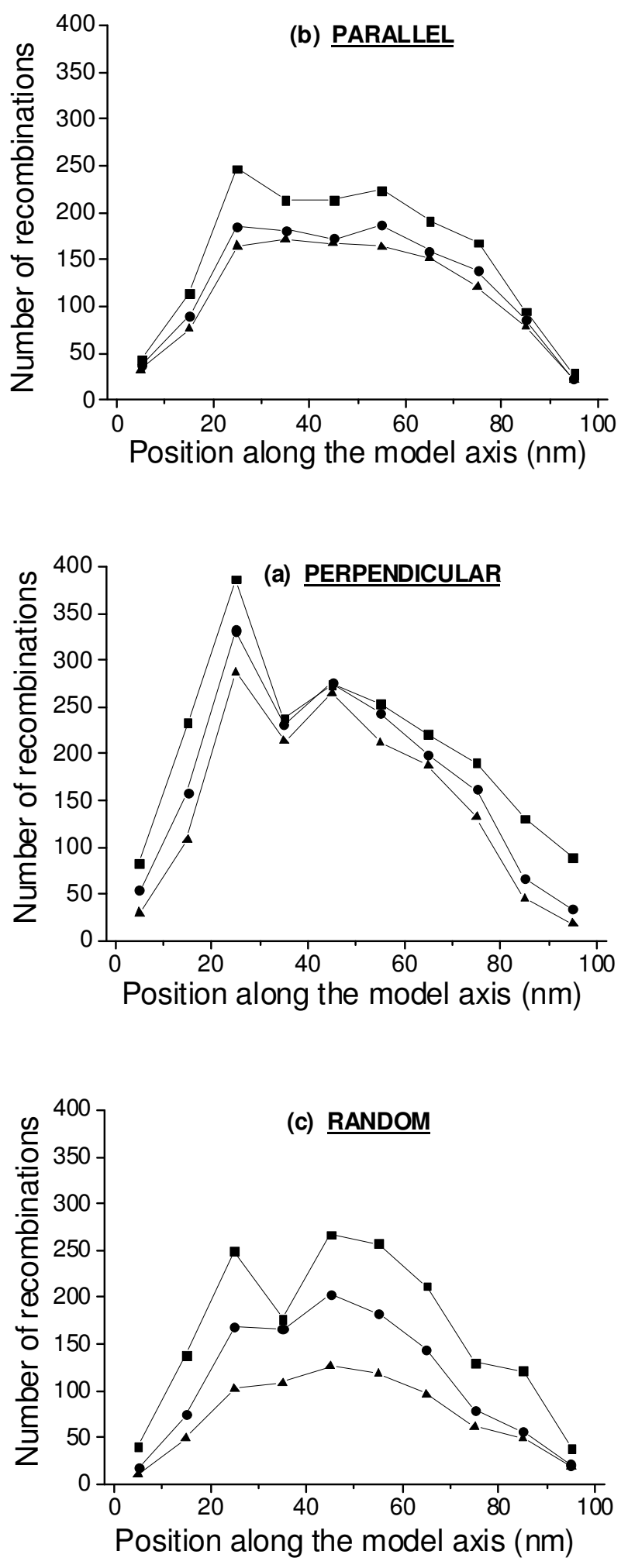